\title{
Do estigma da psicose maníaco-depressiva ao incentivo ao tratamento do transtorno bipolar: a evolução da abordagem em dois veículos midiáticos nos últimos 40 anos
}

From the stigma of manic depression to the encouraging of treatment of bipolar disorder: the evolution of the approach in two

lay media publications over the last 40 years

Daniel Montanini', Cláudio E. M. Banzato

\section{RESUMO}

Objetivo: Investigar a evolução ao longo dos últimos 40 anos da assimilação midiática do transtorno bipolar. Métodos: Foram pesquisadas referências sobre os termos "transtorno bipolar" e "maníaco-depressivo" na revista Veja e no jornal The New York Times e analisada uma amostra de 40 textos de cada veículo. Os textos foram divididos em dois grupos, o primeiro reunindo aqueles datados dos anos entre 1970 e 1999 e o segundo, 2000 a 2009. Resultados: Entre os anos de 1970 e 1999 é mais utilizado o termo "maníaco-depressivo", porém na década de 2000-2009 a expressão "transtorno bipolar" predomina amplamente. Além disso, artigos mais antigos, que utilizam principalmente o termo "maníaco-depressi-

\section{Palavras-chave}

Transtorno afetivo bipolar, psicose maníacodepressiva, classificação diagnóstica. vo", tendem a ser mais estigmatizantes e com menor pretensão informativa. Já artigos mais recentes, em que a expressão "transtorno bipolar" é mais utilizada, tendem a incentivar a busca de tratamento. Conclusão: Sugere-se que a abordagem midiática do transtorno bipolar, comparada ao tratamento recebido pela psicose maníaco-depressiva, é marcada pela maior assimilação do referencial biomédico.

\section{ABSTRACT}

Objective: To examine the evolution over the past 40 years of the midia approach to bipolar disorder. Methods: A search was performed using the terms "bipolar disorder" and "manic-depressive" in the Brazilian magazine Veja and in American newspaper The New York Times. A sample of 40 texts from each source was analyzed. The texts were divided into two groups, one dated of the years between 1970 and 1999 and the second from 2000 to 2009. Results: Between 1970 and 1999 it is most often used the term "manic-depressive", but in the decade of 2000-2009, the term "bipolar disorder" prevails widely. In addition, older articles, which predominantly use the term "manic-depressive" tend to be more stigmatizing and less informative. Recent articles, on the other hand, feature more often the term "bipolar disorder" and tend to encourage treatment seeking. Conclusion: It is suggested that the midia approach to bipolar disorder, in comparison with the one given to manicdepressive psychosis, is characterized by a closer assimilation of the biomedical model.

1 Universidade Estadual de Campinas (Unicamp).

$$
\begin{gathered}
\text { Recebido em } \\
\text { 27/1/2012 } \\
\text { Aprovado em } \\
\text { 08/3/2012 }
\end{gathered}
$$

Endereço para correspondência: Daniel Montanini Departamento de Psicologia Médica e Psiquiatria, Faculdade de Ciências Médicas, Universidade Estadual de Campinas Cidade Universitária "Zeferino Vaz"

Rua Tessália Vieira de Camargo, 126 13083-887 - Campinas, SP

E-mail: danielmontanini@gmail.com 


\section{INTRODUÇÃO}

O transtorno afetivo bipolar ( $T A B)$ é caracterizado por episódios de mania (tipo I) ou hipomania (tipo II) e depressão maior'. Segundo a Organização Mundial de Saúde (OMS), o TAB é uma das 10 condições que causam mais anos de vida vividos com incapacidade. Os últimos relatórios da OMS apontam os transtornos mentais, e entre eles também o afetivo bipolar, como problemas importantes de saúde pública².

A adoção do conceito de TAB surgiu em 1980, com o DSM-III. Classificações diagnósticas anteriores utilizaram nomenclaturas e conceitos diferentes, mas relacionados. Há, portanto, antecedentes históricos relevantes. Esquirol, em 1838, descreveu a alternância entre depressão e mania, sem, contudo, incluir tais manifestações na mesma doença ${ }^{4}$. Jules Falret, em 1851, reuniu os dois quadros numa única entidade, a loucura circular, caracterizada pela alternância da mania e depressão, com intervalos lúcidos ${ }^{4}$. Emil Kraepelin, no fim do século XIX, estabeleceu a importante distinção nosológica entre a chamada dementia praecox, hoje esquizofrenia, e a psicose maníaco-depressiva. Após sucessivas mudanças de nomenclatura e conceito, houve, por fim, a separação entre transtorno bipolar e depressão unipolar, baseada em estudos realizados na década de 1960 por Angst, Perris, Winokur, entre outros pesquisadores ${ }^{4}$.

Percebe-se, ao longo da história da psiquiatria, a existência de várias possibilidades de sistematização dos transtornos, e daí provém a característica dinâmica da classificação diagnóstica. Dessa maneira, é de se esperar que, com as transformações científicas, sociais, políticas e culturais, se discutam e se alterem modelos de classificação. A aproximação entre doença e normalidade é também um fenômeno assimilado pela população leiga, que passa a perceber os diagnósticos médicos e psiquiátricos mais próximos, adotando-os em seu cotidiano ${ }^{5}$. Em um contexto em que as distâncias entre normalidade e doença são encurtadas e suas fronteiras borradas, aparece também a preocupação com a medicalização exagerada dos transtornos mentais, já que os benefícios do tratamento medicamentoso, sobretudo para casos limítrofes, podem ser questionados ${ }^{6}$.

Em uma análise interpretativa dos discursos sobre saúde estampados em matérias de capa de edições de revistas brasileiras, foram encontrados valores comuns que se relacionariam com a concepção de saúde e o contexto social vigentes ${ }^{7}$. Pensando em aspectos específicos de saúde, como é o caso do transtorno afetivo bipolar, faz-se válida a proposta de encontrar e listar os diferentes elementos dos discursos da mídia, já que, de alguma forma, eles são expoentes de concepções de saúde e do próprio transtorno, assimiladas socialmente.

Justificando-se pelas dimensões importantes que o transtorno afetivo bipolar vem assumindo em termos de saúde pública, e tendo como hipótese que o modo de assi- milação cultural de um transtorno psiquiátrico varia ao longo do tempo, assim como se transformam também as classificações diagnósticas, o objetivo do presente estudo foi o de investigar as diferentes maneiras como o transtorno afetivo bipolar e seu antecessor, a psicose maníaca depressiva, foram abordados pela mídia leiga nos últimos 40 anos.

\section{MÉTODOS}

Para análise de expressões midiáticas relacionadas ao transtorno bipolar, foram pesquisadas duas fontes de informações, sendo elas a revista brasileira Veja e o jornal norte-americano The New York Times. Tais veículos jornalísticos foram escolhidos por conveniência, pois ambos disponibilizam digitalmente todo o conteúdo publicado durante o período investigado, o que facilitou sobremaneira a busca e a leitura do material. Não podemos assegurar de forma alguma que nossa amostra seja representativa do universo midiático, porque foram estudados apenas dois veículos (com periodicidade semanal e diária, respectivamente, e enfoque editorial distinto).

Por meio do acervo digital disponibilizado via internet pela revista Veja no endereço http://veja.abril.com.br/acervodigital/, foi realizada a busca pelas palavras-chave "bipolaridade", "bipolar" e "maníaco-depressivo". Das centenas de referências encontradas, selecionamos aquelas publicadas entre os anos de 1970 e 2009. Foram excluídos os textos que não utilizavam os termos em clara referência ao transtorno bipolar ou em expressão dele derivada. Foram retirados, por exemplo, os artigos que utilizavam a palavra "bipolar" para referir o mundo dividido em dois polos na guerra fria e aqueles cuja palavra era utilizada como adjetivo inespecífico que, embora trouxesse a noção de dois polos, não o fizesse considerando um relacionado à depressão e outro à euforia. Após a realização dessa filtragem, restaram 40 textos diferentes, entre artigos, cartas, colunas, entrevistas e notas rápidas. Para o jornal The New York Times, foi realizada a pesquisa por meio da ferramenta Search, disponível em http://www.nytimes.com/. Os termos pesquisados foram "bipolar disorder" e "manic-depressive". Como a amostra final da revista Veja consistiu em 40 textos, optamos por selecionar, de forma aleatória, para o jornal norte-americano The New York Times, uma amostra de mesmo tamanho, ou seja, de 40 artigos. Foi usado para esse fim um programa gerador de números aleatórios, que selecionou 40 números, dentre um intervalo previamente definido, correspondente ao número de referências encontradas no NYT.

Após uma leitura e sistematização prévia dos artigos, foram criadas categorias para análise comparativa entre os anos estudados. Essas categorias incluíam, por exemplo, o termo utilizado, a centralidade do transtorno bipolar para o artigo, a menção ao estigma, as referências positivas ou ne- 
gativas a respeito do transtorno, o incentivo ao tratamento, entre outras. A fim de comparar a expressão midiática do transtorno bipolar em diferentes períodos, os artigos foram divididos em dois grupos assimétricos que contemplavam diferentes cortes temporais, sendo eles de 1970 a 1999 e de 2000 a 2009. O ano de 2000 foi escolhido como ponto de corte, porque se percebeu, na análise de distribuição de frequências (Figura 1), ter sido esse o momento no qual ocorreu um forte incremento no número de artigos que faziam referência à condição estudada.

Para a análise estatística do material, foi utilizado o software Statistical Package for the Social Science (SPSS, versão 13). A pesquisa foi aprovada pelo Comitê de Ética em Pesquisa da Universidade Estadual de Campinas (Unicamp).

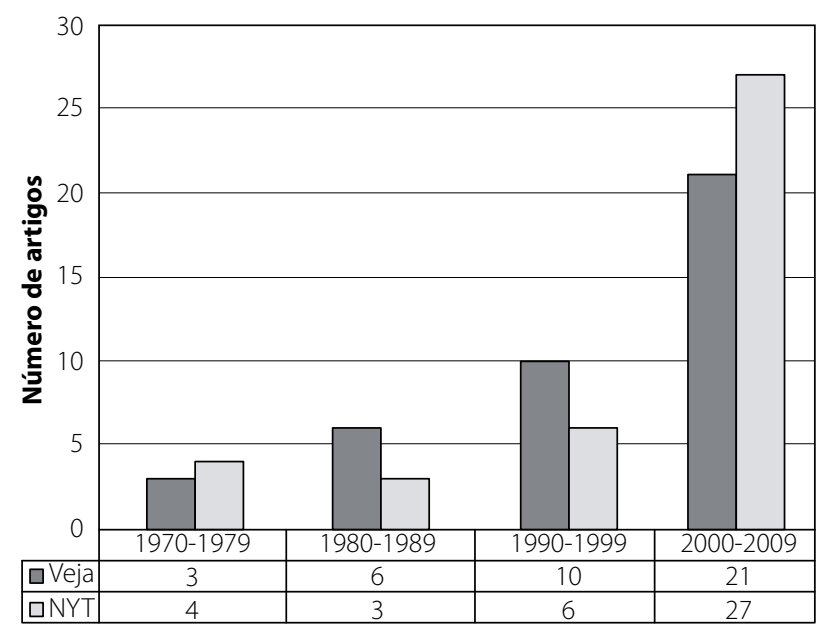

Figura 1. Artigos estudados da Veja e do The New York Times por década.

\section{RESULTADOS}

As referências pesquisadas se distribuíram no tempo de maneira semelhante na revista Veja e no jornal The New York Times. Na Veja, dos 40 artigos encontrados, apenas 3 datavam dos anos entre 1970-1979, já a década de 2000 contava com 21, ou seja, mais da metade da amostra completa para essa revista.

A distribuição temporal dos 40 artigos do The New York Times selecionados aleatoriamente e dos 40 artigos da revista Veja é mostrada na Figura 1. O jornal The New York Times, uma publicação diária e muito mais extensa, naturalmente possui muito mais referências para as pesquisas das palavras-chave relacionadas ao transtorno bipolar entre os anos de 1970 e 2009. O total de artigos encontrados na busca no NYT foi de 1490. Portanto, a amostra estudada de 40 artigos representou 2,68\% do universo pesquisado. A análise da distribuição temporal das referências encontradas para cada termo é in- teressante, pois das 675 referências para bipolar disorder, 582 se encontram na década de 2000, ou seja, $86,22 \%$. Já o termo manic-depressive, que conta com 768 referências no total, foi mais encontrado nas décadas de 1990 (284\% ou 36,94\%) e 2000 (224\% ou 29,16\%). Com esses dados, é possível visualizar um aumento recente no número de publicações de artigos referentes ao transtorno bipolar.

Uma transformação encontrada na apresentação das publicações sobre o tema do transtorno bipolar diz respeito a qual termo é utilizado primordialmente nos diferentes cortes temporais. Percebeu-se que termos derivados de transtorno afetivo bipolar só aparecem com relevância a partir da década de 2000. Na revista Veja, 84,2\% dos artigos de 1970 a 1999 apresentaram apenas algum termo derivado de psicose maníaco-depressiva, e 100\% dos artigos da década de 2000 utilizaram apenas termos relativos a transtorno afetivo bipolar (Figura 2).

Pela análise dos artigos do jornal The New York Times, foi percebido que em alguns deles os termos "bipolar" e "manic-depressive" apareciam como adjetivos, sem qualquer conotação diagnóstica ou médica. Para dar um exemplo, um artigo utilizava "manic-depressive" como adjetivo não para uma pessoa, mas para um concerto musical e, em outro texto, ursos eram chamados de maníaco-depressivos. Esses artigos se mostraram mais prevalentes nas décadas de 1970 a 1999, e dos cinco artigos nos quais os termos pesquisados servem como caracterização sem pretensões diagnósticas, quatro estão entre as décadas de 1970 e 1990.

Comparando a prevalência na revista Veja de abordagens que incentivavam o tratamento do transtorno bipolar, foi encontrada maior proporção de artigos com essa característica na década de 2000. Esse incentivo pode dar-se por meio de uma ênfase ao tratamento (geralmente medicamentoso) ou referências de sucesso no tratamento do transtorno. $\mathrm{Na}$ revista Veja, de 10 artigos que de alguma maneira incentivavam o tratamento, nove datavam da década de 2000. Para o jornal The New York Times, não foi encontrada uma diferença significativa nessa distribuição, e o número de artigos que incentivavam o tratamento do transtorno bipolar, em todos os cortes, era bastante elevado, o que demonstra uma diferença importante em relação à revista brasileira.

\section{DISCUSSÃO}

É importante ressaltar as limitações do presente estudo. Foram pesquisados dois veículos midiáticos bastante diferentes entre si, no que diz respeito à periodicidade, à abrangência e ao enfoque editorial. Não se trata, portanto, de uma amostra representativa da mídia leiga. Outra limitação do trabalho é o pequeno número percentual de artigos do NYT consultados. Por outro lado, a amostra obtida parece ter sido suficiente para capturar certas tendências. 


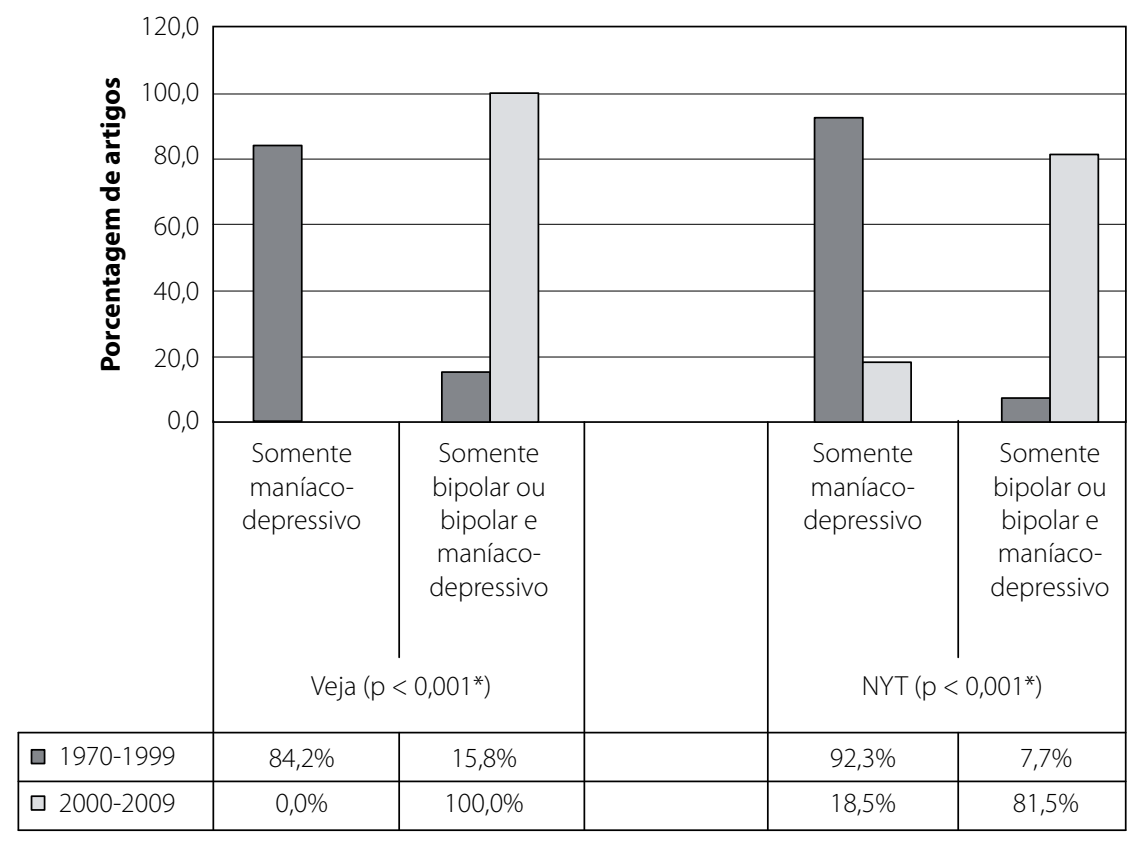

* Pelo teste exato de Fisher

Figura 2. Termo utilizado por corte temporal.

Pela distribuição temporal do número de artigos que tratam do transtorno bipolar, nota-se que é na década de 2000 que se encontra a grande maioria das referências. Essa mesma distribuição demonstra que no intervalo estudado (1970-2009) há um progressivo aumento da atenção da mídia leiga para com o transtorno bipolar, que assim adquire maior visibilidade social.

Conforme já mencionado, o lançamento do DSM-III, manual preconizador do termo "transtorno afetivo bipolar", em substituição à "psicose maníaco-depressiva", data de 1980. Os dados obtidos (Figura 2) no presente estudo, todavia, demonstram que, tanto no jornal norte-americano The New York Times quanto na revista brasileira Veja, a substituição efetiva dos termos acontece apenas na década de 2000. É plausível supor que a assimilação de novas nomenclaturas médicas pela mídia leiga aconteça mais tardiamente, em um segundo momento, após seu uso ser consagrado no campo clínico. Isso explicaria o intervalo de 20 anos entre a publicação do DSM-III e a difusão cultural do novo termo. Vale destacar, ainda, o caráter dinâmico da nomenclatura, isto é, além de identificar objetos e caracterizar aquilo que nomeia, ela deve ser entendida como um dos próprios vetores de mudança ${ }^{8}$. Em outros termos, ela tanto é efeito, na medida em que a adoção oficial de um novo termo representa uma determinada decisão, como causa de modificações, porque sua assimilação dificilmente pode ser prevista por completo a priori. Não é à toa, portanto, que o novo termo, "transtorno afetivo bipolar", possivelmente mais brando e menos estigmatizante do que "psicose maníaco-depressiva", após sua consagração no campo da clínica, passa a ser muito mais utilizado também na mídia leiga em um momento de inflexão da assimilação sociocultural do transtorno.

Termos derivados do TAB aparecem como caracterização sem pretensões diagnósticas significativamente no The New York Times, com mais frequência nas décadas de 1970, 1980 e 1990. Esse resultado demonstra uma particularidade dessa época, em que alusões ao transtorno bipolar adquirem muitas vezes menor ênfase diagnóstica, funcionando como caracterizações por vezes estigmatizantes ou preconceituosas, realizadas sem maior preocupação terminológica.

A década de 2000 reúne a maior parte dos artigos da revista Veja que incentivam o tratamento do transtorno bipolar. Para o jornal The New York Times, entretanto, não é encontrada tal diferença. Além disso, no jornal norte-americano, o número de artigos que incentivam o tratamento do transtorno bipolar é muito mais elevado do que na revista brasileira, em todas as últimas quatro décadas. Esse resultado sugere uma diferença importante entre os dois países. Parece que nos Estados Unidos o incentivo midiático ao tratamento já acontece há mais tempo, sendo no Brasil um fenômeno mais recente.

Os resultados obtidos corroboram a visão de uma expressão midiática atual revelando uma maior ênfase no diagnóstico e tratamento do transtorno bipolar, em relação ao percebido nas últimas três décadas. Além disso, uma abordagem midiática mais comprometida com o referencial biomédico, isto é, aquela que reproduz diagnósticos de forma 
menos estigmatizada, poderia resultar inclusive em maior procura por cuidados médico-psiquiátricos.

\section{CONCLUSÃO}

A maneira como o transtorno afetivo bipolar é difundido pela mídia impressa leiga brasileira e norte-americana se transforma na década de 2000, quando "transtorno afetivo bipolar" ("bipolar disorder") passa a ser o termo predominante, e quase exclusivo, para designar o transtorno, em lugar de "psicose maníaco-depressiva" ("manic-depressive psychosis"), em sintonia com as transformações das classificações diagnósticas oficiais. Sugere-se, portanto, que a assimilação cultural do nome "transtorno afetivo bipolar" refletiu mudanças conceituais e práticas na psiquiatria ocorridas nas últimas décadas. Recentemente, o transtorno bipolar passa a ser mais enfatizado e o seu tratamento médico, mais incentivado no Brasil. O uso da expressão "transtorno bipolar" já não está mais tão relacionado a meras caracterizações descompromissadas e estigmatizantes, como anteriormente, mas relaciona-se cada vez mais com a adoção, por parte da mídia leiga, de um referencial biomédico.

\section{AGRADECIMENTOS}

Este estudo de iniciação científica recebeu apoio do Programa Institucional de Bolsas de Iniciação Científica - Serviço de Apoio ao Estudante da Universidade Estadual de Campinas (Pibic-SAE/Unicamp).

\section{REFERÊNCIAS}

1. Sadock JS, Sadock VA. Transtornos do humor. In: Sadock JS, Sadock VA. Compêndio de psiquiatria: ciências do comportamento e psiquiatria clínica. 9a ed. Porto Alegre: Artmed; 2007. p. 572-629.

2. World Health Organization. The Global Burden of Disease: 2004 Update. Geneva: World Health Organization; 2008

3. Mayes R, Horwitz AV. DSM-III and the revolution in the classification of mental illness. J Hist Behav Sci. 2007;43(4):419.

4. Angst J. Historical aspects of the dichotomy between manic-depressive disorders and schizophrenia. Schizophr Res. 2002;57(1):5-13.

5. Lima RC. Somos todos desatentos? 0 TDA/H e a construção de bioidentidades. Rio de Janeiro: Relume Dumará; 2005. p. 161.

6. Healy D. The latest mania: selling bipolar disorder. PLoS Med. 2006;3(4):e185.

7. Silvestre A. 0 sujeito e o pathos na mídia. Uma análise do discurso sobre saúde nas matérias de capa das revistas Veja e Época [dissertação]. São Paulo (SP): Universidade de São Paulo; 2007.

8. Hacking I. The social construction of what. Cambridge, MA: Harvard University Press; 2000. p. 264. 